\title{
Funktionen des „Zeigens“ bei Freud und Wittgenstein
}

\author{
Markus Klammer, Basel
}

Vorab eine terminologische Klärung: Ich werde „Zeigen“ in zwei Bedeutungen verwenden. Wenn ich sage: „Der Traum lässt sich nicht zeigen“, dann verwende ich „Zeigen“ transitiv und meine: „Auf den Traum lässt sich nicht deuten“. Wenn ich sage: „Die Zeichnung zeigt“, dann verwende ich „Zeigen“ intransitiv und meine: „Die Zeichnung weist auf/gibt zu erkennen.“

\section{1.}

Die „Gespräche über Freud“ sind eine lose Zusammenstellung von Notaten. Sie geben den Inhalt von mindestens vier Gesprächen wieder, die Rush Rees zwischen 1942 und 1946 mit Ludwig Wittgenstein geführt hat. ${ }^{1}$ Darin ist vermerkt, dass Wittgenstein, als er kurz nach 1919 das erste Mal „etwas von Freud" gelesen hatte - es ist nicht vermerkt was -, sich vor Erstaunen aufsetzte. Rhees gibt die Begebenheit in direkter Rede wieder: „Dann las ich [Wittgenstein, M.K.] einige Jahre später zufällig etwas von Freud und setzte mich vor Erstaunen auf. Hier war jemand, der etwas zu sagen hatte. " ${ }^{2}$ Laut

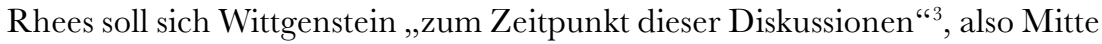
der 1940er Jahre, gar als einen „,Schüler Freuds ${ }^{\varsigma}$ und ,Anhänger Freuds ${ }^{6}$ “4 bezeichnet haben.

Gleichwohl geht es in den „Gesprächen“ um eine Infragestellung der Kernannahmen der freudschen Psychoanalyse. Alle drehen sie sich um die Richtigkeit der analytischen Interpretation des „Materials“, das der Patient während der Kur produziert, also von Träumen, freien Assoziationen und sonstigen Symptomäußerungen. 
Wittgenstein fragt sich:

A. Gibt es überhaupt eine „richtige“ Interpretation innerhalb der Psychoanalyse? Welches sind die Kriterien für die Richtigkeit der Interpretation des Analytikers?

Und damit zusammenhängend:

B. Kann es, wie von Freud behauptet, wirklich nur eine einzige richtige Interpretation geben, sei es eines Traums im Kleinen oder einer Fallstudie im Großen?

C. Wenn die Psychoanalyse wesentlich im Durcharbeiten, d.h. im wiederholten Zurückkommen auf bereits geäußertes Sprachmaterial und dessen ständiger Neubewertung, besteht, worin liegt dann die Stoppbedingung ebenso der freien Assoziationen des Patienten wie der Interpretationstätigkeit des Therapeuten? Mit anderen Worten: Woher weiß ich, dass ich jetzt endlich bei der „richtigen“ Interpretation angelangt bin?

Wittgenstein argumentiert nun nicht entlang der Kriterien Freuds für die prinzipielle Richtigkeit der analytischen Interpretation, sondern nähert sich Problem A über die Sätze B und C: Wenn sich plausibel machen lässt, dass es jedes Mal nicht nur eine einzige richtige Interpretation eines bestimmten Materials gibt bzw. wenn sich zeigen lässt, dass die Kette der Interpretationen dieses Materials prinzipiell unendlich fortgesponnen werden kann, dann bricht das ganze Konzept der „richtigen Interpretation“ der Psychoanalyse, d.h. der interpretierenden Aufdeckung eines primären kausalen Faktors hinter der Pluralität der Assoziationen und Symptome, in sich zusammen. Dabei drücken Satz B und Satz C lediglich verschiedene Aspekte desselben aus: Wenn ich eine unendliche Fortführbarkeit der Reihe der Interpretationen erweise, zeige ich damit zugleich, dass es nicht nur eine einzige „richtige“ Interpretation geben kann. Und umgekehrt: Wenn es mehr als eine Interpretation gibt, gelange ich zu allfälligen weiteren Interpretationen nur durch Fortführung der Tätigkeit des Interpretierens.

\section{2.}

Wittgenstein widerlegt Freud nicht direkt, sondern gibt eine Reihe von Beispielen, die auf die notwendige Pluralität von Interpretationen nach dem Muster der Psychoanalyse verweisen. Dabei geht er von dem Paradigma der Deutung von Träumen aus. Jeder Traum zerfällt nach Freud in einen 
manifesten und einen latenten Anteil. Der manifeste Anteil setzt sich aus Wahrnehmungsresten zusammen, ist bildhaft und ungeordnet, der latente Anteil, Freud sagt auch „Traumgedanken“, ist sprachförmig oder zumindest sprachanalog und dadurch eigentlicher Ansatzpunkt der Deutung. Freud verwendet „Bild“ hier natürlich nicht im Sinne des Tractatus. Was er meint, sind konkrete Formen von Bildlichkeit wie Gemälde, Zeichnungen, Kulissen, Rebusse, die er verwendet, um bestimmte Eigenschaften des manifesten Traums zu verdeutlichen. Die Traumgedanken liegen nach Freud irgendwie „unter“ den manifesten Traumbildern verborgen, abgeschnitten durch die Zensur, und werden von einer einzigen verdrängten Wunschregung organisiert. Gerade diese gilt es in der Interpretation zu rekonstruieren. Die Interpretation schreitet von den Traumbildern zu den Traumgedanken und von diesen zum Traumwunsch. Die Traumbilder an sich sind, einmal abgesehen von der sattsam bekannten Sexualsymbolik, nur schwer verständlich. Erst über die Einfälle des Patienten in der freien Assoziation bildet sich ein Hof möglicher Bedeutungen. Trotzdem scheinen die Bilder des manifesten Traums bereits die Möglichkeit einer Interpretation zu versprechen, sie scheinen auf Deutung hin angelegt zu sein. Freud drückt diesen Umstand im Begriff des „Rebus“ aus. Ein Rebus ist ein Bilderrätsel, das sich gleichermaßen aus sprachlichen und bildlichen Elementen zusammensetzt. Die Wahrnehmungsfragmente des manifesten Traums weisen in der Form ihrer Organisation laut Freud immer schon eine gewisse Sprachförmigkeit auf - wobei Freud Sprachförmigkeit weitgehend mit Deutbarkeit gleichsetzt, denn Deutbarkeit heißt für ihn Kausalisierung, und er geht davon aus, dass sich Kausalrelationen nur innerhalb der Sprache hinreichend deutlich ausdrücken lassen.

Wittgenstein folgt dieser Argumentationslinie weitgehend, wenn er zu Anfang eines Gesprächs von 1943 sagt:

Es scheint etwas in den Traumbildern zu geben, das eine gewisse Ähnlichkeit mit den Zeichen einer Sprache hat. [...] Es mag kein Zeichen dabei sein, das wir als konventionelles Zeichen irgendeines Alphabets erkennen würden, und doch haben wir das starke Gefühl, daß sie irgendeine Art Sprache sein müssen: daß sie etwas bedeuten müssen. ${ }^{5}$

Wie lässt sich dieser Drang des Bedeutens, dieses Bedeuten-Müssen einlösen und in eine konkrete Interpretation verwandeln? Im Sommer 1942 war Wittgenstein auf folgendes Sprachspiel verfallen: Ich male Kritzel an die 
Wand, die in gewisser Hinsicht an eine Schrift erinnern, die aber weder ich noch irgendjemand sonst versteht und die ich „Doodles“ nenne.

Ein Analytiker beginnt dann, mir Fragen zu stellen [...]; und wir kommen zu einer Erklärung, warum ich das tue. Wir könnten dann vielleicht verschiedene Kritzel, die ich gemacht habe, verschiedenen Elementen der Interpretation zuordnen. Und wir könnten dann die Doodles als eine Art von Schrift bezeichnen, als Gebrauch einer Art von Sprache, obwohl sie niemand verstanden hatte. ${ }^{6}$

Wittgenstein beschreibt hier die eigentümliche Nachträglichkeit psychoanalytischen Bedeutens. Erst im Nachhinein, „obwohl sie niemand verstanden hatte“, stellt sich die Bedeutung der Doodles heraus. Es gibt jedoch schlichtweg kein Kriterium, aufgrund dessen ich sagen könnte, dass die von mir gefundene Interpretation die einzige oder die „richtige“ wäre. Die nachträgliche Interpretation, die auf das Gefühl, oder besser, auf die Voraussetzung, des Bedeuten-Müssens folgt, ist auf Pluralität hin angelegt: Es wird vorausgesetzt, dass die Doodles etwas bedeuten müssen, daraus folgt jedoch in keiner Weise, was genau das sein soll, bzw. auf welche Weise sie bedeuten.

Auch das zweite Beispiel Wittgensteins, von 1943, schlägt in dieselbe Kerbe. Es geht von der Bildförmigkeit des manifesten Traums aus. Es handelt von der Tätigkeit der freien Assoziation, lässt sich aber ebenso gut auf die Interpretation des Analytikers beziehen: Man stelle sich das Fragment eines Bildes vor, das es zu vervollständigen gilt. Anstatt raten zu lassen, was es wohl vorstellen könnte, lässt man den Patienten Striche ziehen, wie es ihm gerade in den Sinn kommt, d. h. frei assoziieren. „Das könnte in sehr vielen Fällen ein fruchtbarer Rat sein. Aber es wäre erstaunlich, wenn er immer zu den besten Ergebnissen führte. ${ }^{\text {"7 }}$ Soweit das Gleichnis. Man könnte fortsetzen: Auch die geplante Rekonstruktion des Bildes aus den Strichen des Patienten, also die Interpretation des Analytikers, führt nicht immer zu den besten Ergebnissen, vor allem führt sie nicht zu einem einzigen, alleingültigen, stabilen Ergebnis, weil niemand sagen kann, wann das Bild eigentlich „fertig“ ist. Denn es ist unmöglich zu wissen, wie das ursprüngliche Bild ,zuvor“ eigentlich ausgesehen hat. Es gibt schlichtweg keinen Bildzustand ,vor“ dem Fragment (= dem manifesten Traum), mit dem die Rekonstruktion verglichen werden könnte. Auf das „Urbild“, das vollständige Bild, das die Deutung zu rekonstruieren sucht, kann nicht gezeigt werden. ${ }^{8}$ 
Fassen wir die Hauptkritikpunkte Wittgensteins an Freud zusammen. Sie alle berühren Probleme, die sich nach Wittgenstein einer tiefen Verwurzelung Freuds in physikalischen Paradigmen des 19. Jahrhunderts verdanken. ${ }^{9}$ Wittgenstein kritisiert:

A. Das Bedeuten-Müssen, d. h. die Annahme einer „Kausalität bei der Wirkung von Gefühlen“"10, die er wie folgt umschreibt: „,Es muß ein Gesetz geben', obwohl kein Gesetz gefunden worden ist. "11

B. Die Zentralisierung des Kausalarguments, ${ }^{12}$ d. h. die Annahme einer einzigen richtigen Interpretation, die gleichzeitig auch vollkommen richtig sein muss, um nicht ungültig zu werden. Wittgenstein: „Er wollte eine einzige Erklärung finden, die zeigen sollte, was Träumen bedeutet. [...] Wenn er sich teilweise irrte, dann hätte das für ihn bedeutet, völlig falsch zu liegen $[\ldots]$. “13

Und Wittgenstein kritisiert:

C. Die Realitäts-Prätention des freudschen Deutungsschemas, d.h. die Bindung des Bedeuten-Müssens an die Behauptung, dass es wirklich so gewesen ist, dass so etwas wie eine „Urszene“ wirklich vorgefallen ist:

Ganz bestimmt kann man auf Grund von Freuds Frage [Kann etwas ohne Ursache passieren?, M.K.] nicht die Behauptung aufstellen, daß alles im Traum einen Grund in dem Sinn hat, daß es ein vergangenes Ereignis gibt, mit dem es durch Assoziation auf diese Weise verbunden ist. ${ }^{14}$

Freud entwickelt den Begriff der Urszene in der 1918 erschienen Fallstudie „Aus der Geschichte einer infantilen Neurose“, in der er sich mit der Zwangsneurose des russischen Adligen Sergej Pankejeff auseinandersetzt, der von 1910 bis 1914 in der Berggasse in Behandlung war und als „Wolfsmann“ in die Annalen der Psychoanalyse eingegangen ist. „Urszene“ bezeichnet ein frühkindliches Ereignis, das eine Erwachsenenneurose determiniert, d.h. präfiguriert und in ihren Formen bestimmt. Eine Urszene aktualisiert sich laut Freud unter anderem in den Träumen des erkrankten erwachsenen Individuums, die sie in verkleideter Form wiederholen. Im engeren Sinn handelt es sich bei der Urszene um eine Beobachtung des elterlichen Koitus. Eine solche ist gemäß Freud dem kleinen Sergej passiert: In der Nacht auf seinen vierten Geburtstag, dem russisch-orthodoxen Weihnachtsfest, träumt 
er einen Traum von fünf, sechs oder sieben weißen Wölfen auf einem kahlen Baum. Aufgrund der Einfälle Pankejeffs entlarvt Freud den Traum als Rekonfiguration einer Begebenheit, die der kleine Sergej im Alter von eineinhalb Jahren erlebt haben muss: Die weißen Wölfe werden zu den Eltern, die sich in weißen Gewändern einem nachmittäglichen Liebesspiel hingeben, während der Kleine bewegungslos bei ihnen im Bett liegt. Das angespannte Schauen der Wölfe im Traum spiegelt das Schauen des Kleinen in der Urszene, ebenso wie ihre zum Bild erstarrte Bewegungslosigkeit seine eigene Bewegungslosigkeit wiederholt und die heftigen Bewegungen der Eltern kaschiert. Dem Traum mit vier Jahren liegt laut Freud der Wunsch zugrunde, vom Vater koitiert zu werden, ein Wunsch, der um jeden Preis unterdrückt werden musste und dadurch pathogen wurde.

\section{4.}

Hat Freud nicht gute Gründe dafür, Kausalisierung, Zentralisierung und Realitäts-Prätention anzunehmen? Ist sein System nicht in der direkten und unausgesetzten Beschäftigung mit empirischem Material entstanden und also nicht einfach „Spekulation“"15 oder mythoform ${ }^{16}$, wie Wittgenstein behauptet? Erspart sich Wittgenstein nicht die Mühe, die Interpretationsstrategien Freuds en détail und am Material zu prüfen, um stattdessen mit erfundenen Beispielen zu hantieren? Wenden wir uns also Freuds eigenen Kriterien für die Richtigkeit der Interpretation zu.

In den Aufzeichnungen von Alice Ambrose zu Wittgensteins Cambridger Vorlesungen aus dem Studienjahr 1932/33 findet sich eine Stelle, wo zwischen primären und sekundären Kriterien für die Verifikation einer Aussage unterschieden wird: ${ }^{17}$ Wenn jemand fragt: „Was ist Regen?“, kann man entweder direkt auf den strömenden Regen zeigen oder aber darauf verweisen, dass der Bürgersteig nass ist. Ersteres ist ein primäres Kriterium, letzteres ein sekundäres, das „,die Bedeutung von ,Regen' in nicht so entscheidender Weise ${ }^{\text {“ } 18}$ bestimmt. Ein analoges Sprachspiel könnte man für die Frage: „Hat es geregnet?“ oder „Hat das als Urszene rekonstruierte Ereignis wirklich stattgefunden?" entwerfen. Es ist klar, dass primäre Kriterien mit der transitiven Bedeutung von „Zeigen“ im Sinne von „Deuten auf“ zusammenhängen, bei sekundären Kriterien scheint sich das „Deuten auf“ mit einem Schlussverfahren zu verbinden: „Schau doch! Der Bürgersteig ist nass. Also muss es 
geregnet haben. "Auf die Urszene lässt sich nicht zeigen (im transitiven Sinn), ebenso wenig wie auf alle anderen wichtigen Objekte der Psychoanalyse: das Unbewusste, die Triebe, den latenten Traumwunsch, den psychischen Apparat, etc. Da primäre Kriterien notwendigerweise fehlen, können sie, wenn überhaupt, lediglich aus ihren Wirkungen erschlossen und anhand von sekundären Kriterien verifiziert werden.

Wie sehen diese Kriterien aus? Sie finden sich nicht alleine in der Wolfsmann-Studie, sondern treten über das gesamte psychoanalytische Werk Freuds verteilt und in verschiedenen Zusammenstellungen auf. Die sekundären Kriterien für die Realität des einem Traum, einer Phantasie oder einem Symptomenkomplex zugrunde liegenden Ereignisses, das wir „Urszene“ nennen können, lassen sich in vier Gruppen einteilen.

A. Verifikationsverfahren, die der Psychoanalyse - als Kur und als Wissenschaft - extern sind. Damit ist vor allem die Bestätigung durch ältere Verwandte gemeint, die das Stattgefundenhaben eines rekonstruierten Ereignisses „bezeugen“ können.

B. Verifikationsverfahren innerhalb der psychoanalytischen Therapie. Dazu zählen alle diejenigen Phänomene, die Freud unter den Begriff der „passageren Symptombildung“ fasst: das Nachlassen oder die Veränderung von Symptomen nach der Äußerung einer bestimmten Deutung, das Zunehmen oder Nachlassen des Widerstands oder das Vorkommen „bestätigender Träume“.

C. Verifikationsverfahren, die mit der Person des Analysanden und bestimmten Qualitäten des von ihm produzierten Materials zusammenhängen. Dazu gehört der seltene Fall der direkten Erinnerung des Patienten an die Urszene ebenso wie das unmittelbare Gefühl des Einleuchtens der Deutung, das Wittgenstein so beschreibt: „Man ist verleitet zu sagen ,Ja, natürlich, so muß es sein. “" 19 Weitere Kriterien aus dieser Kategorie sind die übergroße Genauigkeit gewisser Details in Träumen bzw. die übergroße affektive Intensität, mit der diese aufgeladen sind und die nur von einem psychisch folgenschweren realen Ereignis herstammen kann.

D. Verifikationskriterien, die mit der internen Stimmigkeit der gefundenen Deutung zusammenhängen. Freud selbst gebraucht dafür die Metapher des Puzzlespiels:

Gelingt es, den unordentlichen Haufen von Holzplättchen, deren jedes ein unverständliches Stück Zeichnung trägt, so zu ordnen, dass die Zeichnung sinn- 
voll wird, daß nirgends eine Lücke zwischen den Fugen bleibt und daß das Ganze den Rahmen ausfüllt, sind alle diese Bedingungen erfüllt, so weiß man, daß man die Lösung des Puzzles gefunden hat und daß es keine andere gibt. ${ }^{20}$

Man halte dieses Gleichnis neben das Beispiel Wittgensteins von dem zu vervollständigenden Bild. Auf den ersten Blick scheint das Schema ganz ähnlich zu sein: Beide Male ist es um die Rekonstruktion eines bildhaften Ganzen zu tun. Bei Freud jedoch besteht dieses Spiel aus einer finiten Anzahl von Elementen, die sich alle gegenseitig bestimmen. Für ein einzelnes Puzzlestück kann es nur einen ganz bestimmten Platz in der Totalität des Puzzles geben, und dieser Platz wird differentiell von den umgebenden Puzzlestücken bestimmt. Freud setzt hier nichts anderes voraus, als dass das psychoanalytische Material - zumindest der Möglichkeit nach - vollständig und ohne Rest in eine kohärente, quasi-syntaktische Form gebracht werden kann. Dies beinhaltet die Voraussetzung, dass sich überhaupt so etwas wie diskrete „Elemente“ aus dem reichlich fließenden mündlichen Ausgangsmaterial kondensieren lassen. In Wittgensteins Beispiel kommen ebenfalls zu füllende Leerstellen vor, er sieht sie jedoch nicht von der Gesamtheit des bisher Gegebenen bestimmt, sondern betont gerade die (eigentlich unabschließbare) Pluralität der möglichen Lösungen. Freud bleibt auch nicht bei der Vorstellung von Richtigkeit als interner Stimmigkeit der Deutung stehen. Für ihn impliziert die PuzzleAnalogie psychoanalytischer Konstruktion, dass die rekonstruierten Ereignisse wirklich so gewesen sind. Dieses Verfahren hat Wittgenstein in den „Gesprächen" als unzulässig gebrandmarkt. Es handelt sich um eine Schlussfigur, die von ferne an den ontologischen Gottesbeweis erinnert und die Michel de Certeau folgendermaßen umschreibt: „,ça marche', donc c'est réel“. ${ }^{21}$ Mit anderen Worten: Wenn sich das Puzzle zusammensetzen lässt, muss das, was auf dem Puzzle zu sehen ist, eine reale Entsprechung haben.

\section{5.}

Freud gibt keine eindeutige Auskunft, wie die Verifikationskriterien zu handhaben sind. Aus seiner eigenen Praxis wird jedoch ersichtlich, dass er sie, wo immer möglich, miteinander zu kombinieren trachtete, um so ihre „Beweiskraft" zu erhöhen.

Erstaunlicherweise liefert Freud selbst auch gleich die Einwände gegen 
seine kombinierte Kriteriologie: Sämtliche von uns genannten sekundären Kriterien - mit Ausnahme des Kohärenzkriteriums, dem jedoch ohnehin keine „externe“ Beweiskraft zukommt - werden sehenden Auges in ihrer Gültigkeit untergraben:

A. „Externe“ Kriterien: Den Verwandten kann man nicht trauen, sie verfolgen ihre eigenen Interessen, sind selbst oft psychisch krank, streben danach, peinliche Vorkommnisse zu vertuschen, ihre Erinnerungen sind denselben Zensurmechanismen unterworfen wie die Erinnerungen des Patienten. ${ }^{22}$ Außerdem lässt es sich die Analyse zur Ehre gereichen, auf alle externen Stützen zu verzichten. ${ }^{23}$

B. Therapeutische Kriterien: Nicht alle Patienten sind nach der Analyse dauerhaft geheilt, es gibt Rückfälle, Symptome kehren wieder. Von der Analyse eine Totalheilung zu erwarten, wäre verfehlt. Es kann nur darum gehen, das Leben insgesamt erträglich zu machen. ${ }^{24}$ Bestes Beispiel ist der Wolfsmann Pankejeff, der bis zu seinem späten Tod im Jahr 1979 in Wien ein lebenslänglicher Patient verschiedenster Analytiker blieb.

C. Patientenbezogene Kriterien: Selbst wenn sich der Patient an die Urszene zu erinnern glaubt, wie kann ich sicher sein, dass es sich nicht bloß um eine Deckerinnerung handelt, hinter der sich die „richtige“ Erinnerung verbirgt? ${ }^{25}$ Die besondere Deutlichkeit von Details in Träumen oder Phantasien bzw. ihre hohe affektive Aufladung kann ebenso sehr von der Stärke der sie produzierenden Wunschregungen wie von ihrem Realitätscharakter zeugen. ${ }^{26}$ Das unmittelbare Einleuchten einer Deutung beim Patienten kann ebenso gut eine raffinierte Form des Widerstandes sein, um den Therapeuten von der wahren Deutung abzubringen. ${ }^{27}$

D. Bleibt nur das Kohärenzkriterium, das alleine keine Gültigkeit besitzt.

6.

Auch wenn man Freud beim Wort nimmt und seinen eigenen Kriterien für die Verifikation einer psychoanalytischen Konstruktion folgt, gelangt man nicht dahin, an die Notwendigkeit der Richtigkeit dieser Konstruktion zu glauben. Denn Freud durchkreuzt seine eigenen Argumente, indem er zeigt, dass die Kriterien der Richtigkeit ebenso gut Kriterien der Unrichtigkeit oder im besten Fall überhaupt keine Kriterien sein können. Deshalb gebraucht er ein 
zusätzliches Mittel - wir wollen dieses Mittel ,solide Metaphern“ nennen -, um anschaulich zu machen, was eigentlich nicht anschaulich gemacht werden kann: Urszene, Unbewusstes, psychischer Apparat, die Rede vom (manifesten) Traum als Bild oder Rebus, etc. All diese „Phänomene“ sind gar keine Phänomene, weil sie sich nicht zeigen. Nur ihre Wirkungen zeigen sich, es bleibt aber zutiefst fragwürdig, worauf sich diese beziehen. Freuds Verfahren besteht, kurz gesagt, darin, die sekundären Verifikationskriterien (illegitimer Weise, wie der Wittgenstein des Tractatus sagen würde) durch primäre zu ersetzten. Erinnern wir uns an die Stelle aus Wittgensteins Vorlesungen: „Fragt jemand: ,Was ist Regen?‘, kann man auf den strömenden Regen zeigen oder Wasser aus der Gießkanne schütten. Dies sind primäre Kriterien. Daß der Bürgersteig naß ist, ist ein sekundäres Kriterium [...]. " ${ }^{28}$ Anstatt zu sagen: „Der Bürgersteig ist nass, also muss es geregnet haben“, zeigt uns Freud den strömenden Regen, besser gesagt: Er schüttet Wasser aus der Gießkanne.

Im Aufsatz „Freud und der Schauplatz der Schrift“ verwendet Jacques

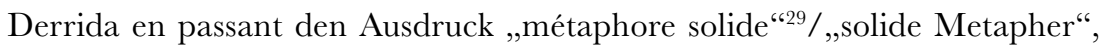
der in der deutschen Übersetzung mit „feste Metapher" ${ }^{\text {"30 }}$ wiedergegeben ist, um den Wunderblock, eines der Modelle Freuds für den psychischen Apparat, zu charakterisieren. Der Wunderblock ist nicht einfach nur eine Metapher des psychischen Apparats, sondern ein Spielzeug und zugleich ein technisches Gerät, das der abendländischen Technikgeschichte zugehört und eine ganz bestimmte Weise des Funktionierens und des Umgangs nahe legt. Auf den konkreten Möglichkeiten der Benützung des Wunderblocks ruhen die Implikationen der soliden Metapher.

Unter einer „soliden Metapher" verstehen wir einen im weitesten Sinn „technischen“ Gegenstand, der für ein an sich unbeobachtbares „Zielmilieu“ einsteht, das bestenfalls und wenn überhaupt aus seinen Wirkungen erschlossen werden kann. Dieser Gegenstand konstituiert ein „Ausgangsmilieu“. Die prinzipiell auf das Zielmilieu übertragbaren Eigenschaften des Ausgangsmilieus werden durch den Möglichkeitsraum vorgegeben, den die konkreten Handhabungen des Apparats aufspannen. Eine solide Metapher zeigt bestimmte Eigenschaften des Zielmilieus, im Sinne von: sie weist sie auf (intransitiv), in den konkreten Möglichkeiten des Umgangs.

Wir unterschieden zwei Arten von soliden Metaphern: Solide Metaphern im übertragenen Sinn und solide Metaphern im wörtlichen Sinn, die wir auch „Archivmaschinen“ (ebenfalls eine Anlehnung an Derrida) nennen wollen. Beide Bedeutungen lassen sich gut am Wunderblock vorführen: Der 
Wunderblock als konkreter Gegenstand des Gebrauchs ist eine „Archivmaschine“. So wie Freud ihn in seiner „Notiz über den ,Wunderblock““ (1925) verwendet, tritt er als solide Metapher im übertragenen Sinn auf. Solide Metaphern im übertragenen Sinn kann man als „Gebrauchsanweisungen" für einen korrespondierenden Apparat verstehen. Der Apparat ist nicht direkt gegeben, die Gebrauchsanweisung richtet den Blick von vorne herein auf ganz bestimmte Funktionsweisen aus. Hätten wir ihn vor uns, wir würden wahrscheinlich noch andere entdecken.

\section{7.}

Freuds Texte sind voll von soliden Metaphern. Dazu zählen unter anderem das Telephon, das Archiv mit seinen Faszikeln" ${ }^{31}$, das „Kinotheater ${ }^{632}$, die Embryologie der Pflanzen ${ }^{33}$, das Fernrohr, das zentrale Nervensystem, das Mikroskop $^{34}$, die Protozoen ${ }^{35}$, die Chirurgie ${ }^{36}$, die Kriegskunst ${ }^{37}$, aber auch Schriftstellerei, Skulptur, Malerei und Zeichnung. Häufiger sind die soliden Metaphern im übertragenen Sinn, die „Gebrauchsanweisungen“, was bei einer textlichen Gattung wie der psychoanalytischen Studie nicht verwundert. Es finden sich jedoch auch Archivmaschinen oder solide Metaphern im wörtlichen Sinn in den Arbeiten Freuds.

Betrachten wir die Seite 604/605 der Erstveröffentlichung der WolfsmannStudie aus der 1918 erschienenen Sammlung kleiner Schriften zur Neurosenlehre. Vierte Folge (Abb. 1, umseitig). Aufgrund von erhaltenen Fahnenkorrekturen aus der Library of Congress in Washington weiß man, dass Freud minutiös in den Prozess der Drucklegung eingegriffen hat. ${ }^{38}$ Aufgabe dieser Doppelseite ist es, den Traum von den Wölfen, der sich in der Therapie als Kern der Zwangsneurose Pankejeffs herausgestellt hatte, darzustellen. Der Traum ist doppelt gegeben: Links als Traumtext in gesperrter Schrift und direkter Rede in der 1. Person Singular und rechts - einzigartig im Euvre Freuds - als Zeichnung des Patienten. Klappt man das Buch zusammen, kommen gesperrter Traumtext und Traumzeichnung genau zur Deckung. Versteht man nun die Doppelseite 604/605 im Besonderen und die Sammlung kleiner Schriften zur Neurosenlehre im Allgemeinen als eine Archivmaschine, lässt sich Folgendes sagen: Die Doppelseite 604/605 zeigt das Ausgangsmaterial der Psychoanalyse. Die solide Metapher der Traumzeichnung steht für den manifesten, bildhaften Traum. Die solide Metapher des Traumtexts ersetzt die mündliche 


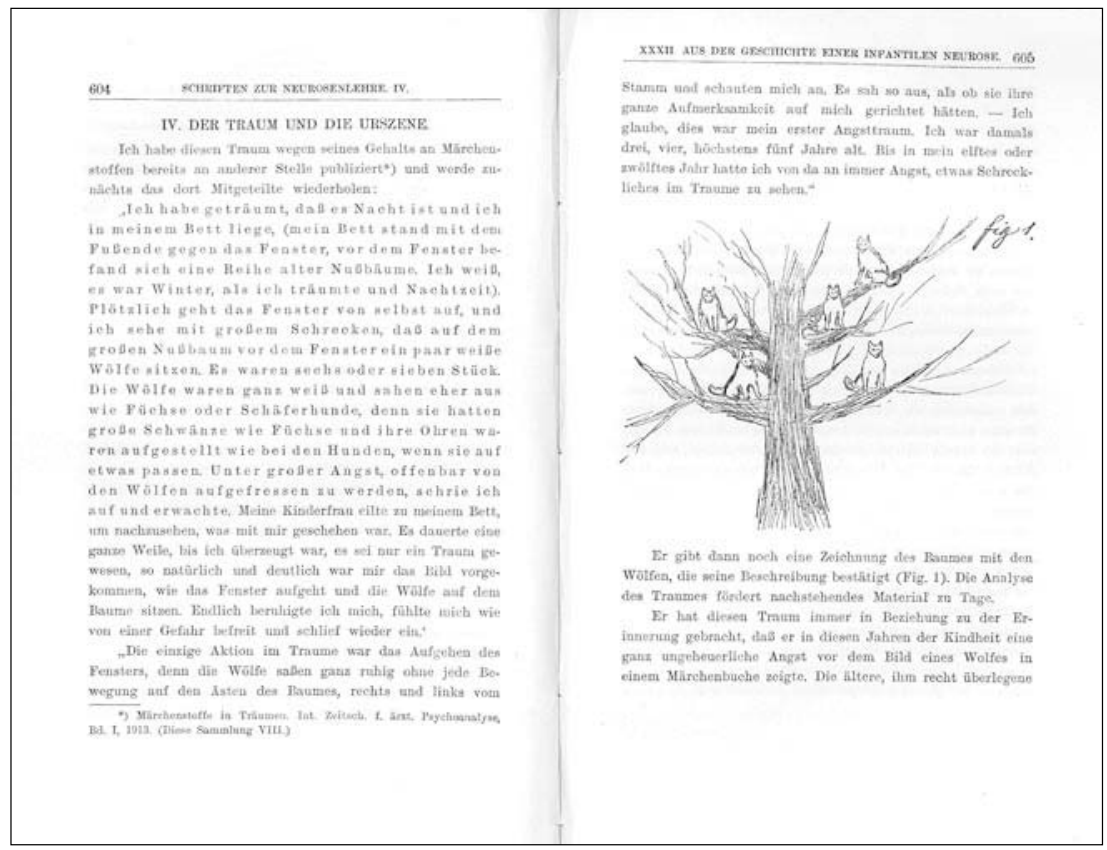

Abb. 1. Wolfsmann-Studie: „Der Traum und die Urszene” (S. 604/605) Sigmund Freud, Sammlung kleiner Schriften zur Neurosenlehre. Vierte Folge, Leipzig/Wien 1918.

Rede des Patienten, die diesen Traum beschreibt und als Ausgangsbasis für alle weiteren Deutungsoperationen dient. Gleichzeitig weist sie auf die vollendete textliche Deutung voraus und zurück auf die latenten Traumgedanken, die den „Schriftraum“39 des Traums bevölkern. Die Deckungsgenauigkeit von Traumtext und Traumzeichnung führt die prinzipielle Relationierbarkeit von Traum und mündlicher Schilderung vor Augen: dass das, was weder sprachlich gegeben ist noch irgendwie (transitiv) gezeigt werden kann, doch prinzipiell in Sprache übertragbar und einer sprachlichen Lösung zugänglich ist. Diese Relationierbarkeit kann niemals bewiesen, sondern nur vorausgesetzt werden. Sie muss sich an soliden Metaphern zeigen (intransitiv). 
Man kann das Problem der Nicht-Zeigbarkeit (transitiv) des Traums auf den Tractatus Wittgensteins beziehen. Dort setzt der Satz als (richtiges oder falsches) Bild eines Sachverhalts der Welt eine bijektive Beziehung der Namen des Satzes und der Gegenstände des Sachverhalts voraus. Als Gegenstände kommen nur raumzeitlich verortbare, ,zeigbare“ (transitiv) Objekte in Frage. Im Unterschied zu Russell, der dem Satz „Der gegenwärtige König von Frankreich ist kahl“ einen Wahrheitswert, nämlich „falsch“ zuschreibt (über die Transformation in: „Es gibt genau einen König von Frankreich, und dieser ist kahl"), hält Wittgenstein diesen Ausdruck schlicht für unsinnig, da der hinweisenden Kennzeichnung „der gegenwärtige König von Frankreich“ kein Gegenstand entspricht. Namen ohne korrespondierende Gegenstände sind ohne Bedeutung. Der Satz, in dem sie auftreten, verfällt als Ganzes dem Unsinn. In dieser Hinsicht sind die Grundvoraussetzungen Freuds allesamt unsinnig, weil sie auf Gegenstände verweisen, die es strenggenommen gar nicht „gibt“. Andrerseits lässt sich eine gewisse Ähnlichkeit zwischen den Gleichnissen und experimentellen Sprachspielen des späteren Wittgenstein und den soliden Metaphern Freuds feststellen. Beide benützten ihre „Zeigemaschinen“ dort, wo keine weiteren Begründungen möglich sind und Voraussetzungen gemacht werden müssen oder wo die Referenz von Namen und Sachverhalten fraglich bleiben muss. Freilich streben sie dabei ganz verschiedenen, um nicht zu sagen, konträren, Polen zu, wie wir anlässlich der Puzzle-Metaphorik Freuds und des Beispiels vom fragmentarischen Bild Wittgensteins gesehen haben.

In $\$ 133$ der Philosophischen Untersuchungen handelt Wittgenstein, wie auch in den „Gesprächen“, von dem Problem des Abbrechens. Es geht jedoch nicht um das Abbrechen der auf Rekursionen hin angelegten psychoanalytischen Interpretationstätigkeit im geeigneten Moment, d. h. bei der richtigen Deutung, sondern um die Schwierigkeit des Abbrechens des Philosophierens. Anders als Freud bindet Wittgenstein den Moment des Abbruchs nicht an ein dem Denken äußeres Ereignis, das zugleich als Ursprung und Lösung des behandelten Problems gesetzt wird, sondern er betont die ganze Willkürlichkeit und Kontingenz, die mit dem Problem des Abbrechens (und wohl auch dem des Beginnens) verbunden ist, so dass man nur von einer Pluralität von Anfängen und Abbrüchen wird sprechen können. 
Die eigentliche Entdeckung ist die, die mich fähig macht, das Philosophieren abzubrechen, wann ich will. - Die die Philosophie zur Ruhe bringt, so daß sie nicht mehr von Fragen gepeitscht wird, die sie selbst in Frage stellen. - Sondern es wird nur an Beispielen eine Methode gezeigt, und die Reihe dieser Beispiele kann man abbrechen. - Es werden Probleme gelöst [...], nicht ein Problem. Es gibt nicht eine Methode der Philosophie, wohl aber gibt es Methoden, gleichsam verschiedene Therapien. ${ }^{40}$ 


\section{Anmerkungen}

1 Vgl. Ludwig Wittgenstein, „Gespräche über Freud“, in Ludwig Wittgenstein: Vorlesungen und Gespräche über Ästhetik, Psychoanalyse und religiösen Glauben, herausgegeben von Cyril Barrett (Frankfurt am Main: Fischer Taschenbuch Verlag, 2005), 60-74.

2 „Gespräche über Freud“, 61.

3 Ebenda.

4 Ebenda.

5 „Gespräche über Freud“, 66. (Hervorh. M.K.)

6 „Gespräche über Freud“, 65. (Hervorh. M.K.)

7 „Gespräche über Freud“, 68.

8 Freilich kann auch auf den manifesten Traum nicht gezeigt werden. Vgl. dazu Abschnitt 6.

9 Vgl. Wittgenstein, „Gespräche über Freud“, 69.

10 „Gespräche über Freud“, 62.

11 Ebenda.

12 Vgl. dagegen das Problem „Warum bestrafen wir Kriminelle?“ auf Seite 72.

13 Wittgenstein, „Gespräche über Freud“, 69.

14 „Gespräche über Freud“, 71.

15 „Gespräche über Freud“, 65.

16 Vgl. „Gespräche über Freud“, 73.

17 Vgl. Ludwig Wittgenstein, Vorlesungen 1930-1935 (Frankfurt am Main: Suhrkamp, 1984), $181 \mathrm{f}$.

18 Vorlesungen 1930-1935, 182. (Hervorh. M.K.)

19 Wittgenstein, „Gespräche über Freud“, 74.

20 Sigmund Freud, „Bemerkungen zur Theorie und Praxis der Traumdeutung“, in Sigmund Freud: Studienausgabe, Ergänzungsband: Schriften zur Behandlungstechnik, (Frankfurt am Main: Fischer Taschenbuch Verlag, 2000), 265.

21 Michel de Certeau, Histoire et psychanalyse entre science et fiction (Paris: Gallimard, 2002), 134.

22 Vgl. Sigmund Freud, „Vorlesungen zur Einführung in die Psychoanalyse“, in Sigmund Freud: Studienausgabe, Bd. 1: Vorlesungen zur Einführung in die Psychoanalyse und Neue Folge (Frankfurt am Main: Fischer Taschenbuch Verlag, 2000), 441-443; Sigmund Freud, „Ratschläge für den Arzt bei der psychoanalytischen Behandlung“", in Sigmund Freud: Sammlung kleiner Schriften zur Neurosenlehre. Vierte Folge (Leipzig/Wien: Hugo Heller, 1918), 411. 
23 Vgl. Sigmund Freud, „Aus der Geschichte einer infantilen Neurose“, in Sigmund Freud: Sammlung kleiner Schriften zur Neurosenlehre. Vierte Folge (Leipzig/Wien: Hugo Heller, 1918), 586 f., Fußnote **).

24 Vgl. Sigmund Freud, „Studien über Hysterie“, in Sigmund Freud: Gesammelte Werke, Bd. 1: Werke aus den Fahren 1892-1899 (Frankfurt am Main: Fischer Taschenbuch Verlag, 1999), $311 \mathrm{f}$.

25 Vgl. Sigmund Freud, „Über Deckerinnerungen“, in Sigmund Freud: Gesammelte Werke, Bd. 1: Werke aus den Fahren 1892-1899 (Frankfurt am Main: Fischer Taschenbuch Verlag, 1999), 529-554.

26 Vgl. Freud, „Über Deckerinnerungen“, 534, 549; Freud, „Aus der Geschichte einer infantilen Neurose“, 623, Fußnote **).

27 Vgl. Sigmund Freud, „Konstruktionen in der Analyse“, in Sigmund Freud: Studienausgabe, Ergänzungsband: Schriften zur Behandlungstechnik (Frankfurt am Main: Fischer Taschenbuch Verlag, 2000), 400.

28 Wittgenstein, Vorlesungen 1930-1935, $181 \mathrm{f}$.

29 Jacques Derrida, „Freud et la scène de l'écriture“, in Jacques Derrida: L'écriture et la différence (Paris: Seuil, 2006), 337.

30 Jacques Derrida, „Freud und der Schauplatz der Schrift“, in Jacques Derrida: Die Schrift und die Differenz (Frankfurt am Main: Suhrkamp, 1994), 346.

31 Vgl. u. a. Freud, „Studien über Hysterie“, $292 \mathrm{f}$.

32 Vgl. u. a. Freud, „Vorlesungen zur Einführung in die Psychoanalyse“, 43.

33 Vgl. u. a. Freud, „Vorlesungen zur Einführung in die Psychoanalyse“, 320.

34 Vgl. u. a. Freud, „Vorlesungen zur Einführung in die Psychoanalyse“, 421.

35 Vgl. u. a. Sigmund Freud, „Das Ich und das Es“, in Sigmund Freud: Studienausgabe, Bd. 3: Psychologie des Unbewußten (Frankfurt am Main: Fischer Taschenbuch Verlag, 2000), 323.

36 Vgl. u. a. Freud, „Vorlesungen zur Einführung in die Psychoanalyse“, 441.

37 Vgl. u. a. Freud, „Vorlesungen zur Einführung in die Psychoanalyse“, 438.

38 Leider sind die entsprechenden Fahnen für die Seite 604/605 nicht erhalten.

39 Sigmund Freud, Die Traumdeutung (Frankfurt am Main: Fischer Taschenbuch Verlag, 1991), 285.

40 Ludwig Wittgenstein, „Philosophische Untersuchungen“, in Ludwig Wittgenstein: Werkausgabe, Band 1: Tractatus logico-philosophicus, Tagebücher 1914-1916, Philosophische Untersuchungen (Frankfurt am Main: Suhrkamp, 2006), 305. (2. Hervorh. M. K.) 